\title{
Assessing the influence of feedback-inclusive rapid prototyping on understanding the semantics of parallel UML statecharts by novice modellers
}

\author{
$\underline{\text { Gayane Sedrakyan }^{\mathrm{a}} \text {, }}$ \\ $\underline{\text { Stephan Poelmans }}{ }^{\mathrm{b},}$, \\ Monique Snoeck $^{\mathrm{c}}$
}

- $\quad{ }^{a}$ K.U.Leuven, Belgium, Faculty of Business and Economics, Department of Decision Sciences and Information Management, Research Center for Management Informatics (LIRIS)

- ${ }^{\mathrm{b}}$ K.U.Leuven, Belgium, Campus Brussel (HUB), Centre for Information Management, Modeling and Simulation - CIMS, Faculty. of Economics \& Business, Warmoesberg 26, 1000 Brussel

- $\quad{ }^{\mathrm{c}}$ K.U.Leuven, Belgium, Faculty of Business and Economics, Department of Decision Sciences and Information Management, Research Center for Management Informatics (LIRIS), Naamsestraat 69, B3000 Leuven, Office number, HOG 03.118

PREPRINT: This paper has been published in Information and Software Technology

Please cite as follows: Gayane Sedrakyan, Stephan Poelmans, Monique Snoeck, Assessing the influence of feedback-inclusive rapid prototyping on understanding the semantics of parallel UML statecharts by novice modellers, Information and Software Technology, Volume 82, February 2017, Pages 159-172, ISSN 0950-5849, http://dx.doi.org/10.1016/j.infsof.2016.11.001.

(http://www.sciencedirect.com/science/article/pii/S0950584916303044) 


\title{
Assessing the influence of feedback-inclusive rapid prototyping on understanding the semantics of parallel UML statecharts by novice modellers
}

\begin{abstract}
Context: UML diagrams are the de facto standard for analysing, communicating and designing software systems, as well as automated code generation. However there is a certain degree of difficulty in understanding a system represented by means of UML diagrams.

Object: Our previous research demonstrates a significant improvement in understanding the structural aspects of a system represented as a UML class diagram when using a feedback-inclusive prototype of a model. This paper extends our previous work with an empirical validation study for the effectiveness of the feedback-inclusive rapid prototyping (FIRP) method, on the comprehension of system dynamics represented as multiple interacting UML statecharts. Because models often combine structural and behavioural views that are highly intertwined, we additionally evaluate the effectiveness of the proposed method with respect to comprehension of the between-view consistency.
\end{abstract}

Method: The FIRP environment was built following the principles of Design Science Research in Information Systems. This study targets the empirical validation of the effectiveness of the proposed technique using an experimental study method. Two experiments were conducted with the participation of 65 final-year master students in the context of different modelling courses from different study programs at KU Leuven using two two-group factorial experimental designs. The effectiveness of the FIRP method was measured by comparing students' performance between the cycles with and without the use of the method, using the understandability (comprehension test results) as the dependent variable and the use of FIRP as the independent variable. Effects from unknown variables were neutralized by means of randomized group compositions. The effectiveness of FIRP was additionally assessed with respect to personal characteristics (age, gender, previous knowledge, self-efficacy) and user acceptance (perceived ease of use, perceived utility, preference, satisfaction).

Results: The findings reveal a significant positive impact of the use of the prototyping technique on students' comprehension of system dynamics represented as multiple interacting statecharts.

Conclusions: The findings provide empirical support for the advantage of the use of FIRP over manual inspection of interacting statecharts. The findings also suggest that the method is suitable for training system's analysis and modelling skills when UML statecharts are involved.

Keywords: conceptual modelling; statechart; model pragmatics; prototyping / simulation; automated feedback; testability of requirements

\section{Introduction}

The success of systems engineering depends heavily on models as the earliest engineering artefacts. Furthermore it is widely recognized that measuring the quality of earliest artefacts is key to developing high-quality systems (Genero, Piattini, \& Chaudron, 2009). Conceptual models currently gain in relevance in the context of the growing importance of compliance between business strategy and ICT realization. Conceptual models are also known for their capability to significantly reduce the complexity of a problem domain by the use of highly abstract representations, thus serving as a communication instrument between business domain and ICT experts in the system design process (Nelson, Poels, Genero, \& Piattini, 2012). Furthermore, "conceptual structures remain the same, from system analysis down through implementation thus making conceptual models the foundation of consistency between the requirements and the final software" (Capretz, 2003). UML became the de facto modelling standard that is widely used for early analysis of the problem domain, documenting the architecture or specifying the detailed design of a system (Lange, 2006). Adoption of UML models has become even more prominent since the introduction of the Model Driven Architecture (MDA) framework and the Model Driven Engineering (MDE) approach to software development (Bézivin, 2006). MDA and MDE recognize that models are the foundation of software system development by focusing on automated code generation from models and hence shifting the focus of software quality assurance from system implementation (software testing) towards system modelling (model verification and validation). However despite the dominance of UML there is a certain degree of difficulty in understanding a system represented by means of UML diagrams (Bavota et al., 2011; Otero \& Dolado, 2004; Siau \& Cao, 2001; Siau, Erickson, \& Lee, 2005). 
Understandability, i.e. a model's ability to be easily understood, has been extensively evaluated in the literature both for static and dynamic UML diagrams. Previous studies pointed out to several contributing factors to comprehension difficulties both by practitioners and juniors. Among them are (1) the level of structural complexity of UML exceeding the limits of human working memory (cognitive load) in terms of the ability for effective information processing (Cruz-Lemus, Genero, \& Piattini, 2008; CruzLemus, Maes, Genero, Poels, \& Piattini, 2010; Erickson \& Siau, 2007; Wilmont, Hengeveld, Barendsen, \& Hoppenbrouwers, 2013); and (2) the lack of comprehension methodologies (Erickson \& Siau, 2007) and, in particular, UML's impreciseness about the combination of interactive, structural and behavioural aspects together in a single model (Gustas, 2010). Furthermore, UML being a general purpose language, it is very "noisy" as it contains a large variety of concepts. Given that only a subset of concepts are relevant given a particular modelling goal, UML's noisiness often results in models that misuse language concepts (Buckl, Matthes, \& Schweda, 2010).

In a teaching context, model comprehension difficulties are additionally associated with the insufficient level of experience of novices and as a result their limited cognitive resources to identify relevant triggers for model verification (Bradley, Paul, \& Seeman, 2006; Damassa \& Sitko, 2010; Schenk, Vitalari, \& Davis, 1998). According to complexity analysis by (Siau \& Cao, 2001) UML class diagram ranks the highest in complexity among the structural diagrams followed by statecharts among the dynamic diagrams (Carbone \& Santucci, 2002; Cruz-Lemus, Genero, Manso, Morasca, \& Piattini, 2009; Cruz-Lemus, Genero, Morasca, \& Piattini, 2007; Genero, Miranda, \& Piattini, 2003) because of their high cognitive and structural complexity (Cruz-Lemus et al., 2008; CruzLemus et al., 2010). In a Delphi study by (Erickson \& Siau, 2007) identifying the kernel of "essential" UML (i.e. diagrams that are highly used) class diagram and statecharts are found to have the highest usability ranks by practitioners and educators from software industry and academic field. Furthermore these are also among the top used diagrams present in the context of educational material such as books, tools, courses and tutorials (with percentages of 100\% (class diagram) and over 96\% (statecharts) (Reggio, Leotta, Ricca, \& Clerissi, 2013) while also being diagrams that support conceptual modelling (Embley \& Thalheim, 2012).

Our previous research demonstrated a significant influence of feedback-inclusive rapid prototyping (also referred to as feedbackenabled simulation) on the comprehension of UML class diagrams. The prototyping method serves as a simulation instrument which allows assessing a model with respect to the desired software system as defined in the requirements. In case of a semantic mismatch the model can be improved through a trial and error correction process by means of modification, regeneration and verification loops. This paper extends our previous work by targeting the empirical evaluation of the influence of the feedback-inclusive rapid prototyping method (FIRP) on understanding the semantics of system behaviour represented by means of parallel UML statecharts (i.e. multiple interacting statecharts). The choice for statecharts is motivated by the fact that UML statechart diagrams have become a core technique for describing the dynamic aspects of a software system (Denger \& Ciolkowski, 2003). UML statechart diagrams are also considered to be one of the most important UML diagrams also recommended for use by practitioners as a starting point for training newcomers to UML (Cruz-Lemus, Genero, Manso, \& Piattini, 2005). While quite some effort has been devoted to defining UML Action Semantics ${ }^{1}$, which later gave rise to the definition of $\mathrm{fUML}^{2}$, the behavioural semantics of UML are still quite complex, defined at a very technical level and therefore not suited for assisting junior modellers in understanding the semantics of interacting statecharts. Conceptual models combine different views of a system - structure and behaviour. Although being different aspects of a system, the structural and behavioural views are highly intertwined. When combining structural and behavioural aspects, i.e. different views of a system in a single model, one should also consider the interrelationship aspects. To our knowledge there are no studies investigating the effects of this dimension and the resulting additional complexity with respect to understandability of a model. In this study we additionally examine the effects of the proposed approach on comprehension of the interplay of explicitly modelled behaviour with implicitly modelled behaviour implied by the structural aspects represented in the class diagram.

Simulation has been known for its benefits for constructing knowledge and skills specifically for complex problem solving tasks requiring experience and has been successfully applied in different domains. Yet, to the best of our knowledge no empirical studies can be found in the context of simulation based learning of statecharts for conceptual modelling. One of the reasons is the major obstacle related to the non-existence of integrated modelling and simulation tools, available to teachers, meaning that in-house development is required in educational institutions. A second significant obstacle relates to the complexity of generating prototypes from models and the risk that generating the prototype may be too time consuming for novices with a limited technical expertise. Yet another challenge is associated with the difficulties of interpreting the outcomes of a simulation (Banks, 1999). With respect to our previous work two contributions are presented: (1) support for simulation-based learning of statecharts in the context of requirements engineering and modelling courses, (2) enhancing the effectiveness of learning behavioural modelling through a cognitive visual

1 http://www.omg.org/cgi-bin/doc?ptc/02-01-09

2 http://www.omg.org/spec/FUML/1.1/PDF/ 
feedback to assist the process of verification/validation of semantic compliance between requirements and a behavioural model. The feedback links the execution results to their causes in a model, thus making the simulation results easy to interpret.

The methodology uses the UML as modelling language, but relies on the concepts of MERODE, an Enterprise Information Systems engineering methodology developed at the university of Leuven. MERODE uses a limited subset of UML in which details irrelevant for conceptual modelling are hidden (Snoeck, 2014), and provides an integrated environment (JMermaid ${ }^{3}$ ) for modelling and one-click FIRP prototype production tool (Sedrakyan \& Snoeck, 2014b) which lowers the required skill-set for model simulation. The work proposes an extension to the techniques previously presented by (Sedrakyan \& Snoeck, 2014b; Sedrakyan, Snoeck, \& Poelmans, 2014; Snoeck, 2014).

The research question is formulated as follows: "Does feedback-inclusive rapid prototyping (FIRP), as a simulation technique, improve a novice modeller's understanding of system dynamics represented by means of a model that consists of multiple interacting statecharts in terms of his/her capability of assessing the model's semantic quality"? An experimental study approach was chosen to evaluate the effectiveness of the proposed FIRP method with respect to understandability. Novices' understanding of a system's behaviour was empirically measured by comparing results of tests without vs. with the use of FIRP using two two-group factorial experimental designs (Trochim, 2000). The findings showed a significant improvement in the novice's capability of understanding system dynamics represented as multiple interacting statecharts when using FIRP simulation. Scientifically, the results contribute to improved knowledge on the cognitive aspects of learning processes in the domain of conceptual modelling and UML statecharts.

The paper is structured as follows. Section 2 describes the learning context, definitions used throughout the paper and the FIRP simulation method. Section 3 describes the methodology for evaluating the proposed FIRP method. Section 4 describes the data analysis and results. Section 5 discusses the contributions and limitations the work. And finally, section 6 concludes the work suggesting possible directions for further research.

\section{Learning context}

The FIRP simulation was developed at KU Leuven within the context of an education innovation project targeting simulation and feedback automation. The tool was used to teach and was tested/validated within the master level course "Architecture and Modelling of Management Information Systems"4 during a period of 6 years involving 600 master level students overall. The course is part of the Management Information Systems program and targets master level students with heterogeneous backgrounds. The goal of the course is to familiarize the students with modern methods and techniques of Object-Oriented Analysis and Design for Enterprise Information Systems, to understand the relation between an information system and the organizational aspects of an enterprise, and to acquire sufficient skills for developing an enterprise model as basis of an enterprise information system. During the course students are involved in hands-on sessions, homeworks, project works for which they are required to translate business requirements (5-15 pages) into conceptual enterprise models as basis for building information systems. The models should include a holistic representation of a system description both in terms of structure (represented as a class diagram) and behaviour (represented as multiple interacting statecharts). The learning environment relies on the use of the modelling tool JMermaid which includes a one-click feedback-inclusive prototyping tool (Sedrakyan \& Snoeck, 2013b, 2014b). During the task completion process students can make use of the integrated FIRP simulation tool within JMermaid to test and validate their models.

Some basic concepts that are used throughout the paper are briefly discussed in the following subsections.

\subsection{The use of statecharts for conceptual modelling}

In this work we refer to conceptual models as object-oriented domain models defined as a conceptual and holistic view of a system, defining its concepts or "entities", their relationships, behaviour and interactions (Embley \& Thalheim, 2012). In line with the principles of object orientation, a conceptual model consists of three complementary views: the object view, the dynamic view and the interaction view (Pastor, Gómez, Insfrán, \& Pelechano, 2001; Snoeck, 2014). The research presented in this paper focuses on the use of statecharts for the high-level specification of object behaviour; i.e., the use of statecharts for conceptual modelling. When using statecharts to define the behaviour of individual domain objects, this amounts to the design of separate cogwheels. In the end however, the behaviour of the global system is like a clockwork that results from the interactions between the different gears, and therefore

3 http://merode.econ.kuleuven.be/mermaid.aspx

4 The description of the course can be found under: http://onderwijsaanbod.kuleuven.be/syllabi/e/D0I71 AE.htm 
understanding the behaviour of multiple interacting statecharts is essential for understanding the behavioural aspects of a conceptual model.

According to the object-oriented paradigm, objects will interact with each other by means of message passing, that is, having one object's methods call another object's features. These interactions are documented by means of collaborations diagrams, the semantics of which are defined separately from the semantics of statecharts. The UML action semantics for concurrency are however only defined for concurrent regions within a single state machine. As a result, understanding the semantics of multiple interacting finite statecharts based solely on UML semantics is far from evident. Even worse, as the semantics of interacting statecharts contain several semantic variation points, different users may even have different understandings of the semantics. In practice, different formalisations have been proposed for UML behavioural semantics based on (amongst others) CSP (Dan \& Danning, 2010), Petri-Nets (Bernardi, Donatelli, \& Merseguer, 2002; Merseguer, Campos, Bernardi, \& Donatelli, 2002), Statemate (Eshuis \& Wieringa, 2000) or LTS (Liu et al., 2013).

The conceptual model proposed by MERODE resolves the complexity of defining the semantics of multiple interacting statecharts by relying for the purpose of conceptual modelling on multi-party interaction rather than interaction by means of message passing.

Multiparty interaction means that when an event occurs (is produced) it can be consumed by many parties. The formal definition of this model of interaction relies on CSP (Dedene \& Snoeck, 1995; Snoeck \& Dedene, 1998). Concretely, when an event is triggered, all transitions enabled for this event will fire, even when belonging to different finite statecharts. Assume for example the domain of a library. When a copy is returned to the library, the user interface will trigger an event 'return'. This event may appear in the statechart of the object type LOAN to trigger the ending of the loan and in the statechart of the object type BOOK, triggering a transition from the state 'unavailable' to the state 'available'. When the event is triggered, all involved objects will respond and each of them will execute the action associated to the transition. MERODE furthermore specifies that reactions to events should be conceived as transactions, meaning that either all involved objects accept the event and execute the associated transition, or none of them does. This means that if one of the involved objects is not in an accepting state, the event will not be consumed by any of the objects. In the given example, assume that an event 'borrow' appears in the statecharts of the LOAN class and the BOOK class. If borrowing the book is not allowed according to the state of the involved book, then also in the loan class the event will not be processed. In MERODE, the object-event table indicates for each event which classes are involved. In order to check for the admissibility of an event, first the set of relevant statecharts can be identified using the object-event table. Upon which each of the statecharts can be checked for acceptance (or not) of the event, given the state of the participating objects.

In the remainder of this text we use the terms parallel statecharts and multiple interacting statecharts interchangeably.

\subsection{Measuring understandability}

In this work understanding of a model is evaluated as the ability to assess the quality of a model. For the definition of model quality, we refer to the quality dimensions of the Conceptual Modelling Quality Framework (Nelson et al., 2012). Within this framework semantic quality (also referred to as external quality) refers to "the level to which the statements in a model reflect the real world in a valid and complete way (feasible completeness, feasible validity)" (Lindland, Sindre, \& Solvberg, 1994). Assessing semantic quality requires reading and understanding the model, and comparing one's understanding of the model with one's understanding of the real world.

\subsection{FIRP simulation of statecharts in the context of conceptual models}

While there is no universal definition of the term simulation, most of the definitions follow the same conventional concept that "simulation is an imitation of a system" (Banks, 1999; Ingalls, 2008) to support decisions with respect to modelling a system. In this work we refer to simulation as the process of generating a prototype from a conceptual model. The definition of a prototype is defined as "fully functional to prove a concept" (Hess, 2012) and as a "type of design language" (Yang, 2005). Within a learning context that includes "testing of a function of a prototype with the purpose to identify potential issues concerned with problem understanding with respect to its design" (Hess, 2012).

As mentioned above, within this work, we use the JMermaid environment with integrated FIRP tool as means to achieve simulation. For the detail of the FIRP simulation environment as well as its implementation the reader is referred to our previous work (Sedrakyan \& Snoeck, 2012, 2013a, 2013b, 2014b; Sedrakyan, Snoeck, \& Poelmans, 2014). The modelling environment allows managing consistency between the views in an automated way (Snoeck, Haesen, Buelens, De Backer, \& Monsieur, 2007; Snoeck, Michiels, \& Dedene, 2003), i.e. each time when entering specifications in one view, specifications that can be derived for other views are 
automatically generated. For example, when an object is created the required creation and ending events are automatically generated. This also allows generating default statecharts containing initial, intermediate and ending states accordingly with transitions using a create-event to transit an object from its initial (non-existing) state to an intermediate "existence" state, and an end-event leading to its final state, i.e. ending a lifecycle of an object. These default statecharts represent the behaviour implicitly resulting from the class diagram. For each class, the modeller can replace the default statechart by a further specified statechart.

\subsection{Understanding of (multiple interacting) statecharts by means of FIRP simulation}

In this section we demonstrate how FIRP simulation is applied for testing/validating a model. An example of an erroneous model about renting an orchid is shown in Fig. 1. The specification states that if an orchid loses its flowers while on location, this needs to be registered and the orchid becomes unavailable for placement on a customer's location. Once the orchid flourishes again, the orchid can be placed at a customer's location again. In the solution the behaviour of the system is modelled as an interaction between lifecycles (statecharts) of the business objects ORCHID and PLACEMENT.

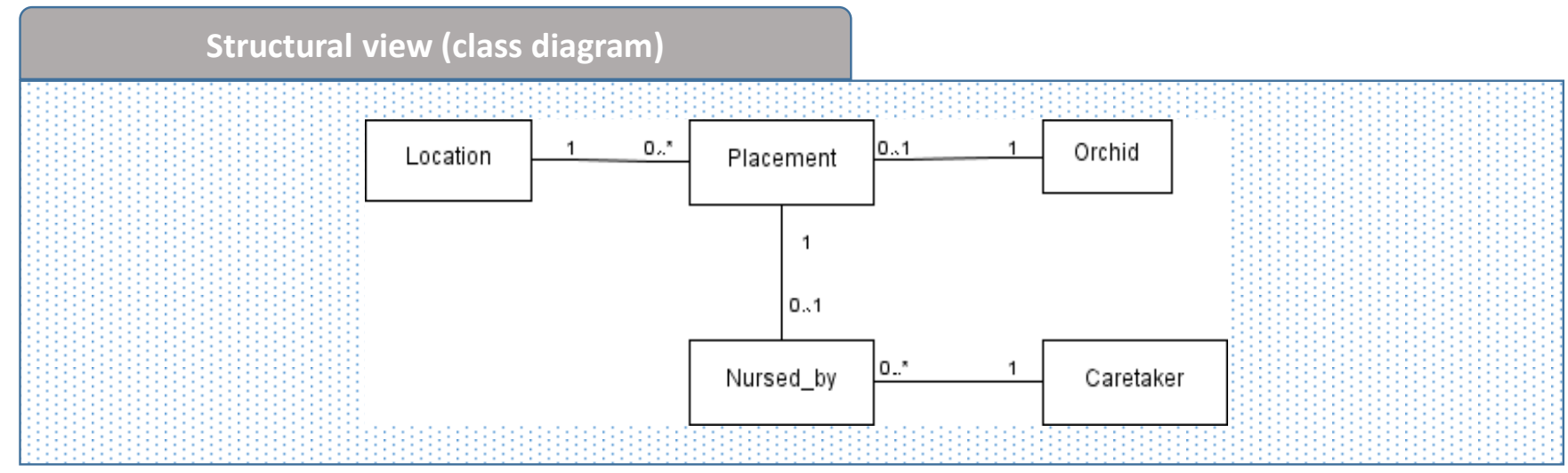

\section{Behavioral view (interacting statecharts)}

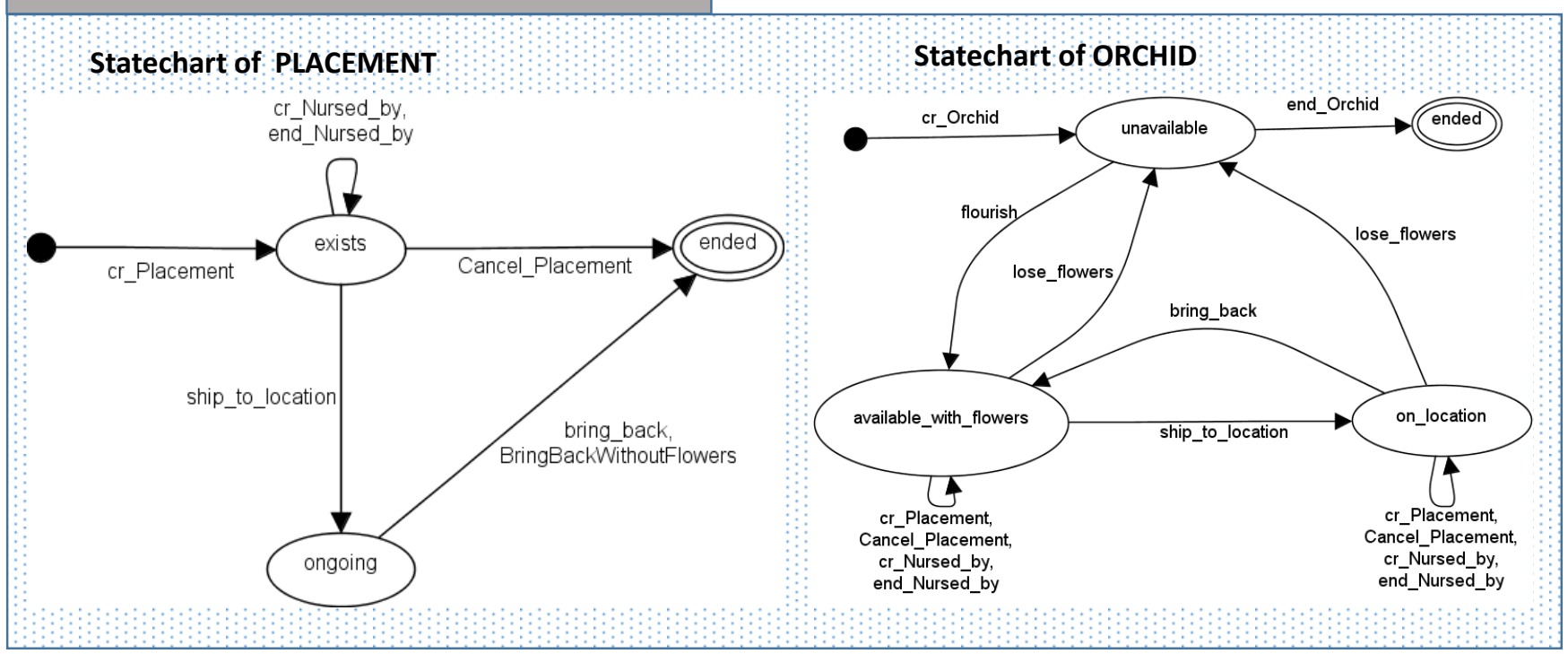

Fig. 1. In the solution the behaviour of the system is modelled as an interaction between the lifecycles (statecharts) of business objects ORCHID and PLACEMENT. 

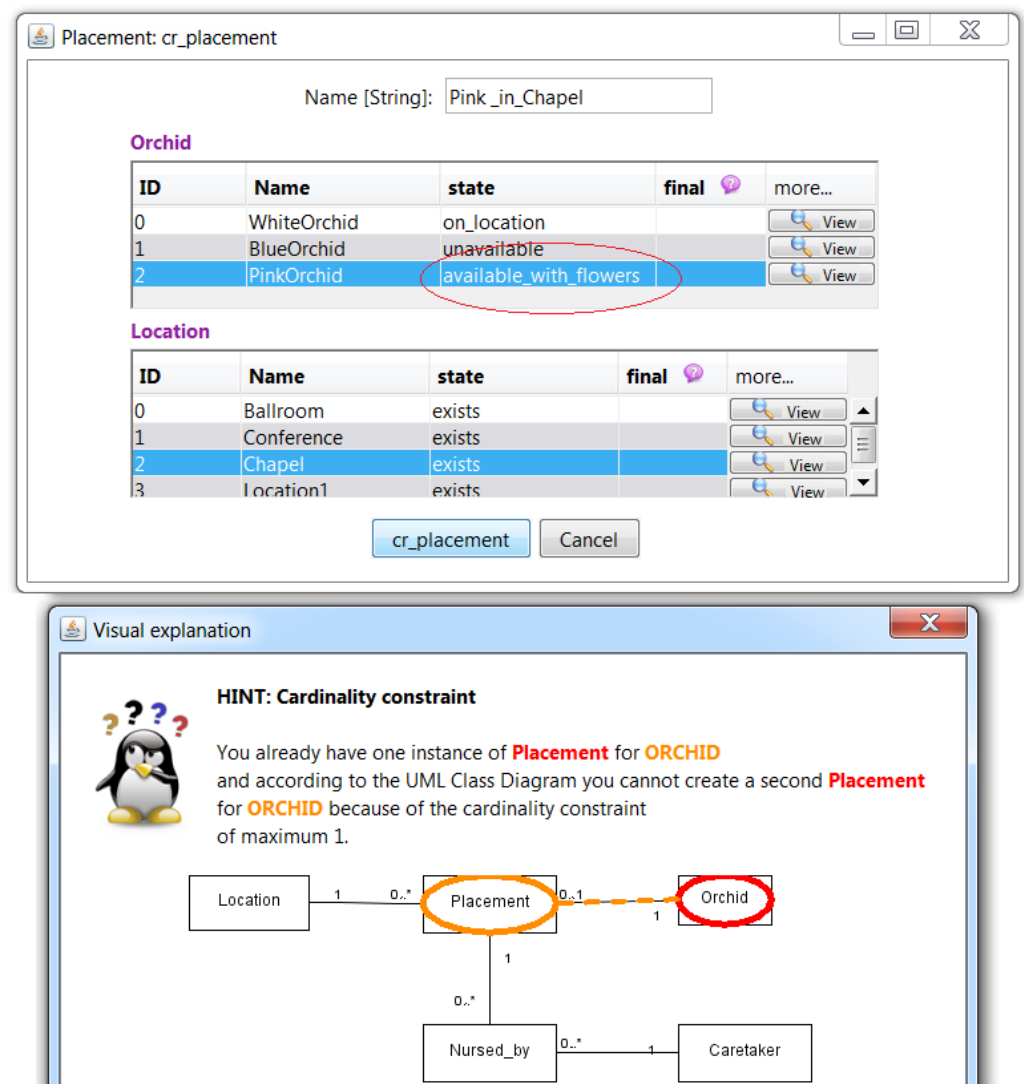

Fig. 2. Event execution refusal due to a cardinality constraint in the class diagram

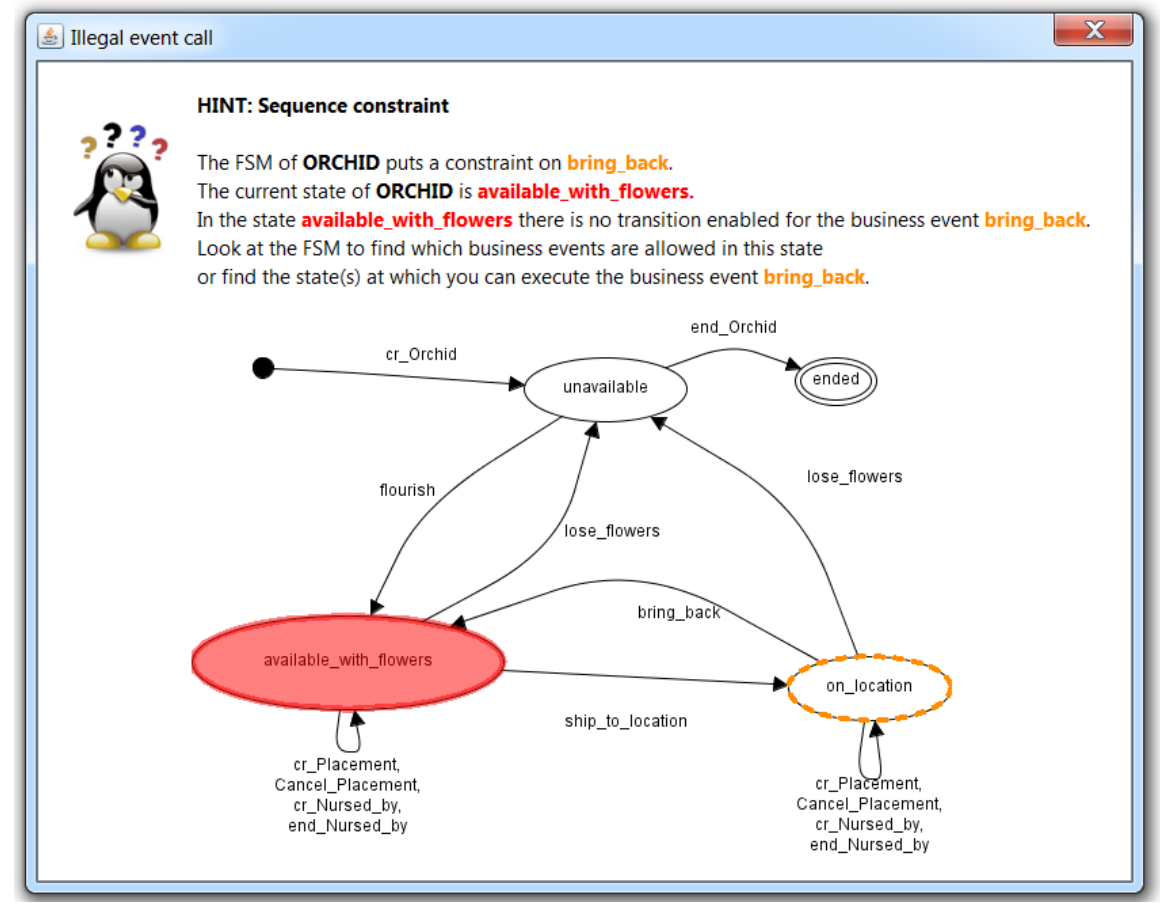

Fig. 3. Event execution refusal due to a sequence constraint (conflicting sequence) between the modelled interacting statecharts for PLACEMENT and ORCHID business objects

Testing the prototype reveals a design error. When registering the loss of flowers by triggering the lose_flowers event for an orchid that is on location, this orchid will indeed become unavailable. After registering its flourishing, the orchid becomes "available_with_flowers" (Fig. 2), yet it turns out to be impossible to create a new placement, as the previous placement was not ended: the event cr_placement is allowed in this state in the statechart of ORCHID, but cardinality constraints in the class diagram 
require a maximum one placement per orchid (Fig. 2). Trying to end the previous placement is not possible either, since according to the statechart of the orchid, the event bring_back is only possible in the state "on_location" and the event bring_back_without flowers is even missing entirely (Fig. 3). The example demonstrates how the complex interplay of cardinality constraints emanating from the class diagram and sequence constraints distributed over several statecharts require advanced mental simulation capabilities to validate a model. The inclusion of feedback in FIRP simulation (Sedrakyan \& Snoeck, 2016) facilitates the interpretation of the causes of the execution failures by means of textual explanations and further extension with graphical visualizations linking the execution results to the parts of the model that causes the failures (Sedrakyan \& Snoeck, 2012, 2013a, 2014b; Sedrakyan, Snoeck, \& Poelmans, 2014).

\section{Research method}

This work extends previous work on the experimental evaluation of FIRP simulation with relation to the understanding of structural aspects. The environment was designed and developed following the principles of Design Science research which among others proposes 2 main guidelines "1.building and 2.(re)evaluating novel artefacts to help understanding and solving knowledge problems" (Hevner, March, Park, \& Ram, 2004). The effectiveness of FIRP simulation (the design artefact) was empirically evaluated by means of two experimental studies: in academic year 2014-2015 the pilot experiment was conducted in the autumn semester on the Leuven campus and a replication experiment was conducted in the spring semester on the Brussels campus. To be able to compare the impact of FIRP on understanding behavioural aspects with the impact on understanding structural aspects (Sedrakyan, Snoeck, \& Poelmans, 2014), the experimental design was kept similar. The experimental design is based on the combination of a two group classical preand post-test control group design with a factorial design (Trochim, 2000). During the experiments students were given a model consisting of a class diagram and multiple interacting statecharts. They were asked to assess whether or not a set of requirements statements were correctly captured by the model (see the appendix). The effectiveness of the FIRP simulation method was assessed by comparing the performance of students on tests without vs. with the use of a FIRP simulation. Furthermore, a cycle rotation comparison was performed (see further explanations in section 3.2 on the experimental design, and section 4 on the analysis of data). We also chose to collect context information with a post-study survey on personal characteristics of students (age, gender, selfefficacy, etc.) (Compeau \& Higgins, 1995; Davis, Bagozzi, \& Warshaw, 1989; Keller, 2009; Poelmans \& Wessa, 2013; Venkatesh, Morris, Davis, \& Davis, 2003). User acceptance (i.e. subjective judgment reflected by satisfaction, preference, perceived usefulness, perceived easiness of use) (Davis et al., 1989; Poelmans \& Wessa, 2013) was chosen to be measured using a Likert-scale technique. Analysis was conducted by means of testing of hypotheses using analysis of variance (Anova, Kruskall-Walis and Wilcoxon), and Kendall's tau coefficients and Cronbach Alpha statistical estimates to measure the correlation of personal characteristics and acceptance variables with the test performance of students.

\subsection{Hypotheses}

We expect that the FIRP simulation would improve a student's understanding of the behavioural aspects of a model, leading to the first hypothesis to test:

H1: FIRP simulation improves the understanding of the behavioural aspects of a model.

To measure this, novices' performance with and without the use of FIRP method were compared (see further details in the analysis section). To ensure the statistical representativeness of the groups/cohorts a pre-experimental test was performed (see further detailed in the section for experimental design) measuring pre-experimental knowledge of the participants. By rotating the cycles in each experiment (see Fig. 4) we intended to measure the group composition and cohort effects (Trochim, 2000).

Even when a didactic environment is proven to be useful, it remains important to ensure that the students also have positive beliefs towards using the didactic environment. Therefore, the students' beliefs towards using the environment were investigated with the following acceptance measures: Preference, Perceived Usefulness, Perceived Ease of Use, and Satisfaction. We expect that the students have a positive stance towards the tool, making it suitable as a didactic tool. This led to the following hypothesis:

H2: The FIRP method is suitable to support its intended utility in a learning context and will likely be accepted by end users (acceptability measured in terms of Satisfaction, Preference, Perceived Usefulness, Perceived Ease of Use, Self-efficacy).

Personal characteristics such as age, gender, as well as acceptance of the tool may affect the results of the experiment (Compeau \& Higgins, 1995; Davis, Bagozzi, \& Warshaw, 1989; Keller, 2009; Poelmans \& Wessa, 2013; Venkatesh, Morris, Davis, \& Davis, 2003). In this study, we test the following hypothesis:

H3: FIRP simulation effects are not affected by personal characteristics. 
The information on both user acceptance (H2) and personal characteristics (H3) were collected using a post-study survey (see Fig. 4). The effects of novices' acceptance beliefs and personal characteristics on test performance was tested using explanatory regression models (see further details in the analysis section).

\subsection{Measurable levels of knowledge with respect to understandability}

Assessing a model's semantic quality requires reading and comparing model statements with the requirement statements. The knowledge of model reading can be evaluated by checking to whether a student makes a correct assessment of the semantic quality of a model with relation to a set of requirements statements. The level of model reading capability can be assessed at different levels:

Level 1: understanding the consistency of a single statechart in isolation, for example, understanding that each state should be accessible from an initial state (forward accessibility), from each state a final state should be reachable (backward accessibility) and that for each event, there should be exactly one transition leading to another state (determinism).

Level 2: reading a transition in the context of a single statechart, for example understanding that reaching a certain state from another state requires an execution of a combination of transitions (sequence of transitions), and that a certain state can be reached from another state by executing of different transitions (alternative transitions).

Level 3: understanding the execution of multiple interacting statecharts based on interaction rules, for example understanding that in the context of event execution all participating objects' lifecycles are affected, i.e. the execution of the event will check the preconditions of each object for that event (required state) and if successful will transit each object to the next state, and that if a precondition of any of the participating objects is not satisfied each object will return to its prior-execution state.

Level 4: understanding the consistency of a behavioural model with the class diagram. This requires understanding the interplay of explicitly modelled behaviour in the (interacting) statecharts with the behavioural constraints implicitly implied by the structural aspects represented in the class diagram. For example, understanding that while a statechart enables an unlimited number of executions of a creation event for some object (e.g. the statechart from order allows creating multiple invoices), a multiplicity constraint in a class diagram may disallow this scenario (an order may have maximum one invoice). Or, understanding that referential integrity rules for a 1-to-many association in a class diagram will assume first a sequence of transitions for ending the lifecycles of all "dependent" objects (on the "many" side of the association), followed by the ending-event in the lifecycle of the referring object (on the "one" side of the association).

\subsection{Experimental design}

Design: Two experimental studies were conducted in the academic year 2014-2015 with the participation of a total of 65 students on the Leuven campus and the Brussels campus. The experiments followed a factorial two group experimental design. According to this design, experiments were conducted with two groups and two cycles within each group. This allows to measure the between-circle learning effects (e.g. performing better in the second experimental cycle because of getting used to a case in the first experimental cycle), as well as the effects from group composition (e.g. prevailing number of better or worse performing students) by analysing the cycle rotation effects for the two groups (Grubišić, Stankov, Rosić, \& Žitko, 2009; Trochim, 2000). The experiments were conducted using two cohorts (pilot and replication study). Within each cohort the participants were randomly assigned to two subgroups (Group1 and Group-2). For each experimental cycle in each group we made use of two different but equivalent experimental cases (test cases with a model and questions) that were altered within each group during the cycles to eliminate the case repetition effects between the cycles. In Group-1 the experiment was started with the use of Case-1 in the first cycle without the use of a FIRP simulation and was continued with Case- 2 in the second cycle with the use of a FIRP simulation. In Group- 2 the experiment was started with Case-1 in the first cycle with the use of a FIRP simulation continued by Case-2 in the second cycle without the use of a FIRP simulation. The design of the experimental setup is shown in Fig. 4. The reversed cycles in the two groups allowed studying case learning effects. In both groups the actual experiment was preceded by a pre-experimental test targeting at the measuring the knowledge of the participants corresponding to levels 1 and 2 (i.e. understanding of basic concepts on statecharts). The experiment itself targeted at measuring model understanding at levels 3 and 4 . 


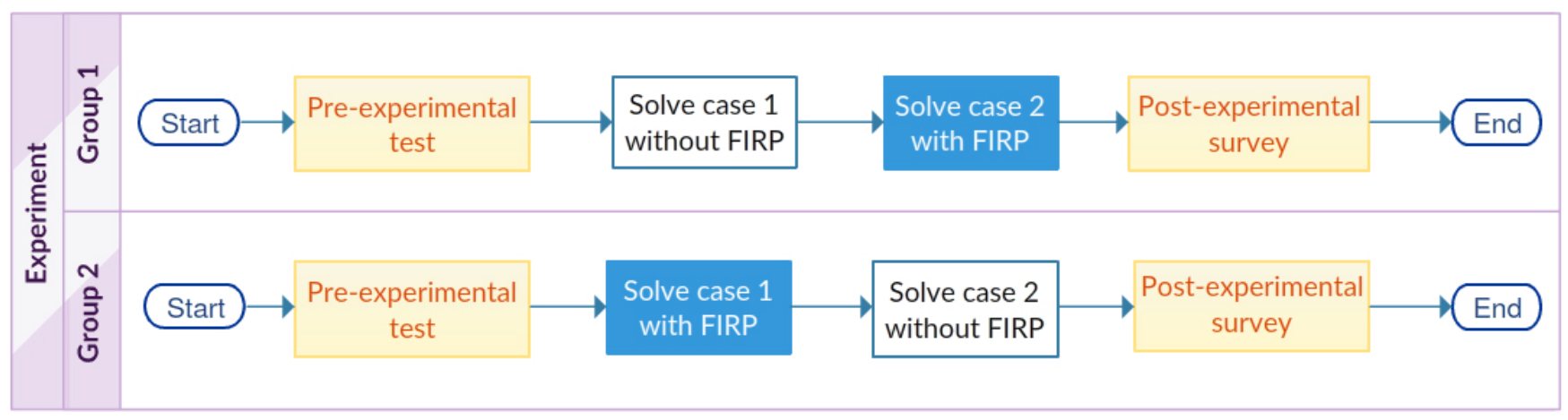

Fig. 4. The experimental design used in the pilot and replication experiments as flowchart diagrams. The experiments combine a classical pre- and post-test control group design with a factorial design (Trochim, 2000) with two groups in each experiment. The blue tasks show where the treatment is applied. The entire experiment was performed once in Leuven and once in Brussels.

The experiments in both groups were followed by a post experimental survey targeting the measurement of extraneous variables. We chose to collect context information with a post-study survey on personal characteristics of students (age, gender, self-efficacy, etc.) (Compeau \& Higgins, 1995; Davis, Bagozzi, \& Warshaw, 1989; Keller, 2009; Poelmans \& Wessa, 2013; Venkatesh, Morris, Davis, \& Davis, 2003). User acceptance (measured by satisfaction, preference, perceived usefulness, perceived easiness of use) (Davis et al., 1989; Poelmans \& Wessa, 2013) was chosen to be measured using a Likert-scale. Analysis was conducted by means of testing of hypotheses using analysis of variance (Anova, Kruskall-Walis and Wilcoxon), and Kendall's tau coefficients and Cronbach Alpha statistical estimates to measure the correlation of personal characteristics and acceptance variables with the test performance of students.

Pilot study: The original experiment was conducted in the autumn semester of the academic year 2014-2015 with the participation of master students $(\mathrm{N}=36)$ from the Leuven campus of KU Leuven. The study was conducted in the context of the course "Architecture and Modelling of Management Information Systems" taught at the Leuven campus with master level students having heterogeneous backgrounds (having non-technical or technical bachelor/master degrees, and also containing working students from ICT industry) from the Master of Information Management program and the Master of Business Engineering: Information Systems. The experiment was conducted in mid-semester after a series of lectures and exercise sessions on data and behaviour modelling.

Replication study: The replicated experiment was conducted in the spring semester of the academic year 2014-2015 with the participation of final year master students $(\mathrm{N}=29)$ subscribed to the course "Design of a Business Information System", at the Brussels campus of KU Leuven. The students had familiarity with data modelling (one semester of study) and were given a lecture and exercise session on behaviour modelling right before the experiment.

Variables: With respect to H1, in our experimental setup the model understanding capability was considered to be the dependent variable. This variable was measured by the test performance, i.e. test score. The scoring was done based on the students' answers about their understanding of a model by testing their ability to assess if the model reflected a particular execution scenario, e.g. "in this model solution, starting from state $\mathrm{X}$, the state $\mathrm{Y}$ in the lifecycle of the object $\mathrm{Z}$ can be reached through the execution of a sequence of events $a, b, c, d, e, \ldots$ ( TRUE/FALSE? )". The students were also required to motivate their responses, i.e. describe the reasons for the outcome (is it successful? why? If not, at which step and why will the execution fail?). 1 point was attributed for each correct response, no point was given for incorrect responses. The test included 5 questions per measurable knowledge level, resulting in a total of 10 questions with a total score between 0 and 10. There were no strict timing requirements for each cycle; however, a maximum of two hours was allowed for completing the entire task (case 1 and 2). The FIRP simulation was considered to be the independent variable. By manipulating the independent variable, i.e. applying the treatment, the effects on the dependent variable, i.e. model understanding, was measured. In Fig. 4, the blue tasks show where the treatment was applied, and the blue arrows indicate how effectiveness can be measured as a relative differential advantage between groups and cycles.

In this study we also chose to measure several other (extraneous variables) with respect to the intended utility of the FIRP simulation. Because "acceptance of a tool is another factor that can affect a learner performance" (Venkatesh et al., 2003) we chose several constructs from established technology acceptance models to be controlled in our experiments (Davis et al., 1989; Poelmans \& Wessa, 2013; Wixom \& Todd, 2005) such as perceived ease of use and perceived usefulness based on (Davis et al., 1989), preference and satisfaction (Bourgonjon, Valcke, Soetaert, \& Schellens, 2010; Ives, Olson, \& Baroudi, 1983; Wixom \& Todd, 2005). Three questions were specified for each of these variables in a post-study survey. Because the effectiveness of a tool can vary for different users, we also controlled personal characteristics (such as gender, age, self-efficacy,...). 


\subsection{Validity of the experimental design}

The internal validity was ensured by means of strict experimental design. The two experiments were designed according to a twogroup factorial experimental design (Trochim, 2000), i.e. two groups and two experimental cycles within each group. As indicated in the previous section this design allows controlling between-circle learning effects (e.g. learning effects from repetition of experimental cases, the order in which treatment was applied), as well as the effects from group composition by analyzing the effects of reversed cycles between the two groups (Grubišić et al., 2009; Trochim, 2000). The potential effects from unknown variables were minimized by means of randomized assignment and the use of control groups to measure the baseline for unknown variables. Normality of pre and post test performance data was tested, globally, i.e. the entire population, per cohort and per group to ensure the statistical equivalence of groups. Subsequently, corresponding statistical tests for measuring treatment effects matching the results were performed.

To minimize the learning case effects two different but equivalent learning cases were used. To minimize distortion effects resulting from answers based on domain knowledge rather than based on the diagrams (e.g. if a case relates to a banking system a student may revert to his/her knowledge of real world situations while completing a task) a (meaningless) alphabetical representation has been used, e.g. object X, Y, Z, W... and events A, B, C, D .... An equivalent but slightly modified case was used for each cycle within each group both in the pilot study as well as in the replication study (different letters, altered graphical arrangement). To avoid straightforward recognition of similarity of the cases by the students, we also shuffled the questions between the cases (see Appendix 1: Experiment cases). For the same reason we reversed some of the questions to vary between the cases (questions formulated to accept a positive answer were rephrased to accept a negative answer and vice versa, e. g. "the sequence of events $\mathrm{X}$, Y, $\mathrm{Z}$, W is required to transfer the object A from state 1 to state 4 " rephrased into "the sequence of events $\mathrm{X}, \mathrm{Y}, \mathrm{Z}, \mathrm{W}$ is not successful for transferring the object A from state 1 to state 4"). The sets of questions were also rearranged in each case.

To ensure the statistical representativeness of the each group a pre-experimental test and a post-study survey were performed. The preexperimental test aimed at ensuring the basic modelling skills of participants. The post-experimental survey aimed at collecting the context information about participants and their subjective judgments about the learning effectiveness of the simulation method using a questionnaire with Likert scales for the answers (see Appendix 2: Evaluation questionnaire). Relevant constructs from the literature related to personal characteristics (age, gender, previous knowledge, self-efficacy) and user acceptance (perceived ease of use, perceived utility, preference, satisfaction) that could potentially affect the experiment results were chosen to be controlled using a post-survey method. Subsequently, to ensure the representativeness of the experimental groups a demographic analysis was performed.

A replication method was used to ensure the validity and reliability of the results. External validity was ensured by means of experiments using different cohorts from different campuses of KU Leuven, i.e. different groups of students from two different courses in different semesters, also taught by different teachers.

\section{Data analysis}

In the following subsections we present the results of the data analysis, namely: participants demographics, test performance, within and between group analysis as well as correlations of the performance with participants' personal characteristics and acceptance measures.

\subsection{Participants}

The demographics of the participants are summarized in Table 1. The participants were final year master students with heterogeneous backgrounds from two different master courses from the Leuven and Brussels campuses at KU Leuven, taught by different instructors in 2014-2015. The first cohort that participated in the pilot study was composed of 36 students of the autumn semester from the Leuven campus; the second cohort of the replication study was composed of 29 students of the spring semester from the Brussels campus. In total 65 participants were engaged in the pilot and replication experiments. 


\begin{tabular}{|c|c|c|}
\hline & Leuven cohort & Brussels cohort \\
\hline \multicolumn{3}{|l|}{ Gender } \\
\hline Male & $68 \%$ & $55 \%$ \\
\hline Female & $32 \%$ & $45 \%$ \\
\hline \multicolumn{3}{|l|}{ Age } \\
\hline Minimum & $22 y$ & $22 \mathrm{y}$ \\
\hline Maximum & $42 y$ & $36 y$ \\
\hline$<=25$ & $80 \%$ & $79 \%$ \\
\hline$>25<=35$ & $11 \%$ & $20 \%$ \\
\hline$>35$ & $9 \%$ & $1 \%$ \\
\hline \multicolumn{3}{|c|}{ Prev. knowledge } \\
\hline Extensive & $11 \%$ & $6 \%$ \\
\hline Moderate & $38 \%$ & $38 \%$ \\
\hline Little & $34 \%$ & $28 \%$ \\
\hline No & $17 \%$ & $28 \%$ \\
\hline
\end{tabular}

Table 1: Summary of demographics

\subsection{Performance test results}

The first step of the analysis compares the mean scores between the experimental cycles of the entire group and of each cohort separately. Scores range on a scale from 0 to 10. Mean scores without the use of FIRP are denoted by $\overline{\mathrm{X}}$, mean scores with the use of FIRP are denoted by $\overline{\mathrm{X}}_{\text {FIRP. }}$. We measured the effectiveness of FIRP as the relative differential advantage, denoted by $\overline{\mathrm{X}}_{\text {RelDiff. }}$ Because of the non-normality of the scores' distributions and the limited size of the subgroups, non-parametric statistical tests were applied to compare the mean scores of experimental cycles within each cohort as well as the entire population. The Kruskal-Wallis test was used to compare means between groups; and the Wilcoxon signed-rank test was used to test paired scores. These methods are the equivalents of the independent t-test and the paired t-test respectively. We also filtered away a small number of cases with empty responses and those without motivation in order to eliminate the effect of false negative and false positive answers.

\begin{tabular}{l|l|l|l|l}
\hline Cohort & $\overline{\mathbf{X}}$ & $\overline{\mathbf{X}}_{\text {FIRP }}$ & $\overline{\mathbf{X}}_{\text {RelDiff }}$ & P-value \\
\hline Entire (Leuven \& Brussels) & 4.59 & 8.36 & 3.77 & 0.000 \\
\hline Pilot experiment (Leuven campus) & 4.08 & 8.14 & 4.06 & 0.000 \\
\hline Replication experiment (Brussels campus) & 5.25 & 8.64 & 3.39 & 0.000 \\
\hline
\end{tabular}

Table 2: Analysis comparing mean scores (without vs with the use of FIRP)

The first step resulted in the analysis shown in Table 2. A cohort effect in relative advantage was discovered showing that in the pilot experiment the relative differential advantage $\left(\overline{\mathrm{X}}_{\text {RelDiff }}=4.06\right)$ was higher than in the replicated experiment $\left(\overline{\mathrm{X}}_{\text {RelDiff }}=3.39\right)$. The results however provide evidence for the positive effect from the use of the FIRP simulation method in both experiments at a significant confidence level of $\mathrm{p}=0.000$ thus supporting our first hypothesis $(\mathrm{H} 1)$.

\subsection{Within/between group analysis}

In the second step of the analysis we performed a Wilcoxon signed-rank tests within groups comparing the test scores with and without the use of FIRP between the two cycles for that group (shown in Table 3). We could observe significant positive effect in both groups of each cohort by comparing the test scores between experimental cycles within each group, thus confirming our first hypothesis (H1).

\begin{tabular}{c|l|l|l|l|l}
\hline \multicolumn{1}{c|}{ Cohort } & Group & $\overline{\mathbf{X}}$ & $\overline{\mathbf{X}}_{\text {FIRP }}$ & $\overline{\mathbf{X}}_{\text {RelDiff }}$ & P-value \\
\hline \multirow{2}{*}{ Pilot study (Leuven participants) } & Group-1 & 2.61 & 8.72 & 6.11 & 0.000 \\
\cline { 2 - 6 } & Group-2 & 5.55 & 7.55 & 2 & 0.004 \\
\hline \multirow{2}{*}{ Replication (Brussels participants) } & Group-1 & 5 & 8.87 & 3.87 & 0.001 \\
\cline { 2 - 6 } & Group-2 & 5.75 & 8.41 & 2.66 & 0.005 \\
\hline
\end{tabular}

Table 3: Within group analysis (Group-1 started without the FIRP)

We could detect group effects in relative advantages in the test scores: the results in group-1, starting without the FIRP in both cohorts, $\left(\overline{\mathrm{X}}_{\text {RelDiff }}=6.11\right.$ in the pilot experiment and $\overline{\mathrm{X}}_{\text {RelDiff }}=3.87$ in the replication experiment $)$ are significantly higher than in 
Group-2 $\left(\overline{\mathrm{X}}_{\text {RelDiff }}=2\right.$ in the pilot experiment and $\overline{\mathrm{X}}_{\text {RelDiff }}=2.26$ in the replication). This difference is mainly due to a higher score in the non-FIRP cycles for groups-2. ( $\overline{\mathrm{X}}=5.55$ and 5.75 in the pilot and replication). Having reversed the treatment in Group-2, by using the FIRP simulation in the first cycle, we could still detect a positive effect both in our pilot experiment $\left(\overline{\mathrm{X}}_{\text {RelDiff }}=2\right)$ and replication $\left(\overline{\mathrm{X}}_{\text {RelDiff }}=2.66\right)$.

In the next step of the analysis we compared the performance that the two groups achieved in the similar cycles of the experiment. Before the analysis we performed F-tests to check the differences between Group-1 and Group-2 for variances. Because of the nonnormality of distributions the nonparametric Kruskal-Wallis analyses were applied to compare the scores. The results shown in Table 4 revealed a significant difference in terms of the differential relative advantage when comparing the scores of the cycle (with the use of FIRP) of Group-1 with the scores of cycle (with the use of FIRP) of Group-2. Furthermore, in the pilot experiment the difference in performance of Group-1 $\left(\overline{\mathrm{X}}_{\text {difference }}=1.17, \mathrm{p}=0.003\right.$ ) was found to be significantly higher, which can again be an indication of a learning (maturation) effect from the use of the case in the previous cycle of the experiment. The difference is however non-significant in the replication experiment $\left(\overline{\mathrm{X}}_{\text {difference }}=0.29, \mathrm{p}=0.182\right)$. The Kruskal-Wallis test for performance comparison for Group-2 comparing test results without the use of FIRP simulation with the results of the same cycle (without FIRP) of Group-1 revealed a significant difference of $\overline{\mathrm{X}}_{\text {difference }}=2.94$ for the pilot experiment. This suggests a maturation effect from using FIRP in the previous cycle within Group-2. In the replicated experiment the difference in mean scores was found to be non-significant $\left(\overline{\mathrm{X}}_{\text {difference }}=0.54 ; \mathrm{p}=\right.$ 0.531). This discrepancy in significance between the Leuven and Brussels cohort can be explained by the fact that the students who participated in the replication experiment (campus Brussels) had the advantage of an intensive training which included a demonstration of validation benefits with the use of simulation and a small exercise session right before the experiment. They might therefore have benefited from practiced knowledge that was still active in their working memory as opposed to the participants of the initial experiment, who only followed a lecture on basics of behaviour modelling and had to find the simulation benefits themselves by exercising during the experiment.

The maturation effects that we detected in the results of Table 3 and Table 4, is supported by an additional qualitative analysis of the students' answers. For instance, analysing the students' responses and explanations in Group-2 provided in the experimental cycle without the use of FIRP simulation, both in the pilot and replication experiments, revealed wording similarities with the FIRP feedback which was different from the way students formulated their explanations in the first cycle of experiments in Group- 1 when no FIRP was provided yet. In the first cycles (without the use of FIRP) in Group-1 both for the pilot and replication experiments, students formulated the explanation of their responses in their own words (e.g. "the lifecycle of the object allows this sequence" or "it is impossible to execute event $\mathrm{X}$ at this step") when trying to mentally execute the scenarios of test questions. In Group- 2 of both cohorts, we observed that the explanations in the second cycles (without FIRP assistance) of the experiment following the first cycles (with FIRP assistance) differed by containing typical wordings from the FIRP feedback (e.g. "this sequence will be refused: event $\mathrm{X}$ is not enabled as a transition in state $\mathrm{Y}$ in the statechart of $\mathrm{Z}$ object"). This shows that the use of FIRP simulation in the previous cycle of the experiment shapes the perception of the learners when answering the test questions without FIRP. While this suggests that the tool can have a persisting effect for knowledge retention, such effect should however be measured with a longitudinal study or experimenting with a revised design which was beyond the scope of this study.

\begin{tabular}{l|r|l|l|l|l}
\hline \multicolumn{1}{c|}{ Cohort } & Experiment Cycle & Group-1 & Group-2 & $\overline{\mathbf{X}}_{\text {RelDiff }}$ & P-value \\
\hline \multirow{2}{*}{ Pilot study (Leuven participants) } & $\overline{\mathrm{X}}$ & 2.61 & 5.55 & 2.94 & 0.003 \\
\cline { 2 - 6 } & $\overline{\mathrm{X}}_{\text {FIRP }}$ & 8.72 & 7.55 & 1.17 & 0.003 \\
\cline { 2 - 6 } & $\overline{\mathrm{X}}_{\text {difference }}$ & 6.11 & 2 & 4.11 & 0.000 \\
\hline \multirow{2}{*}{ Replication (Brussels participants) } & $\overline{\mathrm{X}}$ & 5 & 5.54 & 0.54 & 0.531 \\
\cline { 2 - 6 } & $\overline{\mathrm{X}}_{\text {FIRP }}$ & 8.67 & 8.38 & 0.29 & 0.182 \\
\cline { 2 - 6 } & $\overline{\mathrm{X}}_{\text {difference }}$ & 3.87 & 2.84 & 1.03 & 0.414 \\
\hline Entire (Leuven \& Brussels participants) & $\overline{\mathrm{X}}$ & 3.69 & 8.70 & 5.01 & 0.008 \\
\hline & $\overline{\mathrm{X}}_{\text {FIRP }}$ & 5.55 & 7.90 & 2.35 & 0.000 \\
\hline & $\overline{\mathrm{X}}_{\text {difference }}$ & 5.00 & 2.35 & 2.65 & 0.000 \\
\hline
\end{tabular}

Table 4: Between group analysis

The next step of the analysis targeted at testing for cohort effects between the experimental cycles. Kruskal-Wallis tests showed no significant differences in relative differential advantages between the cohorts (insignificant p-values shown in Table 5). From within/between group analysis results we can conclude that even though limited group effects were observed, overall these effects do not diminish the significant and positive effect of the FIRP simulation. 


\begin{tabular}{l|l|l|l|l}
\hline Experiment cycle & Pilot experiment (Leuven) & Replication experiment (Brussels) & $\overline{\mathbf{X}}_{\text {RelDiff }}$ & P-value \\
\hline$\overline{\mathrm{X}}$ & 4.08 & 5.25 & 1.17 & 0.095 \\
\hline$\overline{\mathrm{X}}_{\text {FIRP }}$ & 8.14 & 8.64 & 0.50 & 0.222 \\
\hline$\overline{\mathrm{X}}_{\text {difference }}$ & 4.05 & 3.29 & 0.76 & 0.300 \\
\hline
\end{tabular}

Table 5: Between cohort analysis

\subsection{Correlation of performance and acceptance variables}

The next step of the analysis targeted the construction of statistical descriptives for the acceptance measures as well as computer selfefficacy (the results are shown in Table 6). Confirmatory factor analysis was performed with each scale item modelled as a reflective indicator of its theorized latent construct.

\begin{tabular}{l|l|l|l|l|l|l|l}
\hline & N & Mean & $\begin{array}{l}\text { Std. } \\
\text { Deviation }\end{array}$ & Min & Max & Cronbach Alpha & Factor Loadings /item \\
\hline Perceived usefulness & 63 & 4.78 & 1.23 & 1.00 & 6.00 & 0.79 & $0.86 ; 0.91 ; 0.73$ \\
\hline Perceived ease of use & 63 & 4.68 & 0.98 & 3.00 & 6.00 & 0.77 & $0.83 ; 0.85 ; 0.80$ \\
\hline Self-efficacy & 60 & 4.45 & 1.11 & 1.00 & 6.00 & 0.78 & $0.80 ; 0.70 ; 0.76 ; 0.72$ \\
\hline Preference & 63 & 4.78 & 1.20 & 1.00 & 6.00 & 0.89 & $0.94 ; 0.90 ; 0.88$ \\
\hline Satisfaction & 62 & 4.77 & 0.92 & 2.00 & 6.00 & 0.89 & $0.92 ; 0.89 ; 0.91$ \\
\hline Feedback* & 63 & 5.58 & 0.89 & 1.00 & 6.00 & $/$ & $/$ \\
\hline * measured with 1 item
\end{tabular}

Table 6: Indicators for acceptance and computer self-efficacy

The reliability and validity of the results were assessed using a Cronbach Alpha statistical estimate (Segars \& Grover, 1993). Reliability with Cronbach Alphas of at least 0.77, and factor loadings of 0.7 (Hair, Black, Babin, Anderson, \& Tatham, 2006) was found. Average scores above 4.68 on a 6-point Likert-scale reflect positive perceptions. In particular, the FIRP method was accepted with a mean score above 4.68 , perceived usefulness and preference for use with a mean score of 4.78 . The highest score was attributed to the feedback (mean score above 5.58), reflecting a very positive belief. Using regression analysis, no impact of acceptance variables on the differential relative advantage (with vs without the use of FIRP) was identified. The results thus confirm the validity of our third hypothesis (H2).

\subsection{Correlation of performance and personal characteristics}

The next step of the analysis targeted at correlating the test performance with the gender of participants ( 23 female, 40 male students $35.4 \%$ and $63.1 \%$ respectively, with 1 missing value) by means of Anova and Kruskall-Wallis. No significant differences could be detected in the test performance both with and without the use of FIRP, the differential scores with-without, and the acceptance measures, ease of use and satisfaction. For preference and usefulness, a slight but significant gender difference was detected. The male and female mean scores on preference are 4.44 and 5.11 respectively; the male and female means for usefulness equal 4.48 and 5.5. Thus, female students seem to favour the prototype slightly more than their male colleagues.

We can conclude that despite the limited influence of personal characteristics, overall these effects do not diminish the significant and positive effect of the FIRP simulation, thus providing support for $\mathrm{H} 3$.

\section{Discussion}

Our analysis reveals a significant positive impact of the FIRP method on novices' understanding of system behaviour represented as multiple interacting statecharts (Cfr. H1). The analysis also shows that novices have positive beliefs towards using the didactic environment (Cfr. H2), suggesting that the method will likely be accepted by end users in a learning context. Furthermore, the results of our analysis also suggest that the effectiveness of the method is not affected by personal characteristics such as gender, previous knowledge, self-efficacy, etc. (Cfr. H3), suggesting that the use of the method in a teaching context will likely lead to improved model comprehension and thus learning outcomes. In general, we conclude that the method is preferable over manual inspection of models in a learning/teaching context. 


\subsection{To guide further research in this domain the contributions and limitations of this study are briefly discussed.Contributions}

The results of the work contribute to the research on cognitive aspects of modelling with respect to the dimension of the understandability of behavioural models. The work extends previous research specifically for the understandability of the execution semantics of a set of parallel interacting statecharts in the context of conceptual modelling. The work provides empirically supported results showing that in a learning context the FIRP simulation method has a significant advantage over manual inspection and mental execution of multiple interacting statecharts. We also witness a retention effect, i.e. for group 2 the capabilities obtained with the use of FIRP method persisted even when the method was no longer applied in the second cycle of the experiment. The method thus has a positive influence on cognitive mechanisms behind understanding the dynamics of a system modelled by means of multiple interacting statecharts. The strict experimental design allowed revealing limited effects of group composition and personal characteristics such as previous knowledge and gender. However, the findings show that these effects do not diminish the significant and positive effect from the use of the FIRP method on understanding (interactive) statecharts compared to manual inspection. The FIRP method presented in this study also contributes to novel computer assisted learning practices (European Commission, 2013) allowing the construction of profound knowledge based on experience rather than reading/lecturing and manual exercising alone as traditionally applied in courses for conceptual modelling.

\subsection{Limitations}

The study is limited to the use of interacting statecharts in the context of one particular technique, namely conceptual modelling, as well as to the courses described in the study. We also observed a limited number of false positive and false negative responses in the answers of participants, in particular for the questions that contained a negative wording, i.e. the motivation of a student's answer in fact indicated the opposite of the provided answer. For example, a student answers "FALSE" to a statement "sequence is not successful" while his/her motivation "because the execution will fail at step X" indicates that (s)he actually meant "TRUE" as an answer. We had to manually correct such answers, i.e. "TRUE" was reversed to "FALSE" and vice versa. Another possible limitation of the study to consider is the fact that the case questions were formulated as test scenarios (specific execution sequences). This may have led to a higher learning effect compared with an experiment in which students would have had to figure out testing scenarios (possible combinations of events) themselves. A final potential limitation of the study is that the observation of the effectiveness of the FIRP method is limited to a learning context. A small scale exploratory test in which the rent-an-orchid scenario described in section 2 was proposed to a group of 9 business analysts with an average professional experience of 11 years, revealed that only two of them with respectively 20 and 10 years of experience were able to detect the flaw in the model by means of manual inspection. The other 7 business analysts, with 4 to 20 years of experience, only detected the problem after using FIRP. Expanding the empirical evaluation beyond conceptual modelling and performing a full scale experimental evaluation with the involvement of more experienced practitioners would contribute to higher reliability and generalizability of the results.

\section{Conclusions}

The work presents an easy and intuitive approach by means of FIRP simulation for improving the student's understanding of the semantics of (multiple interacting) statecharts. The work extends our previous research which observed a significant positive effect from the use of FIRP simulation with respect to understanding the structural aspects of a system represented by means of UML class diagram (Sedrakyan, Snoeck, \& Poelmans, 2014). Compared to these previous results, in this study we observed a much higher relative advantage of the use of the FIRP simulation with respect to understanding the behavioural aspects of a system represented by means of multiple interactive statecharts. We attribute this relative higher advantage to the set-up of the current study where students were provided with concrete test scenarios (also for the without FIRP test) compared to the set-up of our previous studies, which followed a requirement statement based testing approach, and in which students had to identify the testing scenarios themselves. Our findings show that the FIRP method is effective in training novices in the context of requirements analysis and conceptual modelling courses. Among the possible extensions of the work an empirical evaluation of the FIRP method with more experienced practitioners are planned. Since the environment contains a logging functionality allowing the observation of the user's interactions within the environment observing testing behaviour could provide more insights on cognitive mechanisms behind the understanding of conceptual models and UML diagrams (Sedrakyan, 2016; Sedrakyan, De Weerdt, \& Snoeck, 2016; Sedrakyan, Snoeck, \& De Weerdt, 2014). Such insight could be used to suggest improved tool assistance, e.g. test automation (Sedrakyan, 2016; Sedrakyan \& Snoeck, 2014a). The empirical evaluation of the effectiveness of the FIRP method with respect to understanding UML diagrams with more advanced concepts such as inheritance (Sedrakyan \& Snoeck, 2015) could be yet another direction for future research. 


\section{References}

Banks, J. (1999). Introduction to simulation. Paper presented at the Proceedings of the 31st conference on Winter simulation: Simulation-a bridge to the future, Volume 1.

Bavota, G., Gravino, C., Oliveto, R., De Lucia, A., Tortora, G., Genero, M., \& Cruz-Lemus, J. A. (2011). Identifying the weaknesses of UML class diagrams during data model comprehension Model Driven Engineering Languages and Systems (pp. 168-182): Springer.

Bernardi, S., Donatelli, S., \& Merseguer, J. (2002). From UML sequence diagrams and statecharts to analysable petri net models. Paper presented at the Proceedings of the 3rd international workshop on Software and performance.

Bézivin, J. (2006). Model driven engineering: An emerging technical space Generative and transformational techniques in software engineering (pp. 36-64): Springer.

Bourgonjon, J., Valcke, M., Soetaert, R., \& Schellens, T. (2010). Students' perceptions about the use of video games in the classroom. Computers \& Education, 54(4), 1145-1156.

Bradley, J. H., Paul, R., \& Seeman, E. (2006). Analyzing the structure of expert knowledge. Information \& Management, 43(1), 7791. doi: 10.1016/j.im.2004.11.009

Buckl, S., Matthes, F., \& Schweda, C. M. (2010). A meta-language for EA information modeling - state-of-the-art and requirements elicitation Enterprise, Business-Process and Information Systems Modeling (pp. 169-181): Springer.

Capretz, L. F. (2003). A brief history of the object-oriented approach. ACM SIGSOFT Software Engineering Notes, $28(2), 6$.

Carbone, M., \& Santucci, G. (2002). Fast\&\&Serious: a UML based metric for effort estimation. Paper presented at the Proceedings of the 6th ECOOP workshop on quantitative approaches in object-oriented software engineering (QAOOSE'02).

Compeau, D. R., \& Higgins, C. A. (1995). Computer self-efficacy: Development of a measure and initial test. MIS Quarterly, 19(2).

Cruz-Lemus, J. A., Genero, M., Manso, M. E., Morasca, S., \& Piattini, M. (2009). Assessing the understandability of UML statechart diagrams with composite states-A family of empirical studies. Empirical Software Engineering, 14(6), 685-719.

Cruz-Lemus, J. A., Genero, M., Manso, M. E., \& Piattini, M. (2005). Evaluating the effect of composite states on the understandability of UML statechart diagrams Model Driven Engineering Languages and Systems (pp. 113-125): Springer.

Cruz-Lemus, J. A., Genero, M., Morasca, S., \& Piattini, M. (2007). Using practitioners for assessing the understandability of UML statechart diagrams with composite states Advances in Conceptual Modeling-Foundations and Applications (pp. 213-222): Springer.

Cruz-Lemus, J. A., Genero, M., \& Piattini, M. (2008). Using controlled experiments for validating uml statechart diagrams measures Software Process and Product Measurement (pp. 129-138): Springer.

Cruz-Lemus, J. A., Maes, A., Genero, M., Poels, G., \& Piattini, M. (2010). The impact of structural complexity on the understandability of UML statechart diagrams. Information Sciences, 180(11), 2209-2220.

Damassa, D. A., \& Sitko, T. (2010). Simulation Technologies in Higher Education: Uses, Trends, and Implications. EDUCAUSE Center for Analysis and Research (ECAR), Research Bulletins.

Dan, L., \& Danning, L. (2010). Towards a formal behavioral semantics for UML interactions. Paper presented at the Information Science and Engineering (ISISE), 2010 International Symposium on.

Davis, F. D., Bagozzi, R. P., \& Warshaw, P. R. (1989). User acceptance of computer technology: a comparison of two theoretical models. Management science, 35(8), 982-1003.

Dedene, G., \& Snoeck, M. (1995). Formal deadlock elimination in an object oriented conceptual schema. Data \& Knowledge Engineering, 15(1), 1-30.

Denger, C., \& Ciolkowski, M. (2003). High Quality Statecharts through Tailored, Perspective-Based Inspections. Paper presented at the Euromicro Conference, 2003. Proceedings. 29th.

Embley, D. W., \& Thalheim, B. (2012). Handbook of Conceptual Modeling: Theory, Practice, and Research Challenges: Springer.

Erickson, J., \& Siau, K. (2007). Can UML Be Simplified? Practitioner Use of UML in Separate Domains. Paper presented at the Proceedings of the 12th Workshop on Exploring Modeling Methods for Systems Analysis and Design (EMMSAD'07), held in conjunction with the 19th Conference on Advanced Information Systems (CAiSE'07), Trondheim, Norway.

Eshuis, R., \& Wieringa, R. (2000). Requirements-level semantics for UML statecharts: Springer.

European Commission, E. U. (2013). Innovative teaching and learning Opening up education: Innovative teaching and learning for all through new technologies and open educational resources. Communication from the commission to the European parliament, the council, the European economic and social committee and the committee of the regions: European Commission.

Genero, M., Miranda, D., \& Piattini, M. (2003). Defining metrics for UML statechart diagrams in a methodological way Conceptual Modeling for Novel Application Domains (pp. 118-128): Springer.

Genero, M., Piattini, M., \& Chaudron, M. (2009). Quality of UML models. Information and Software Technology, 51(12), 1629-1630.

Grubišić, A., Stankov, S., Rosić, M., \& Žitko, B. (2009). Controlled experiment replication in evaluation of e-learning system's educational influence. Computers \& Education, 53(3), 591-602.

Gustas, R. (2010). Conceptual modeling and integration of static and dynamic aspects of service architectures Ontology, Conceptualization and Epistemology for Information Systems, Software Engineering and Service Science (pp. 17-32): Springer.

Hair, J. F., Black, W. C., Babin, B. J., Anderson, R. E., \& Tatham, R. L. (2006). Multivariate data analysis (Vol. 6): Pearson Prentice Hall Upper Saddle River, NJ. 
Hess, T. A. (2012). Investigation of Prototype Roles in Conceptual Design Using Case Study and Protocol Study Methods. Clemson University.

Hevner, A., R., March, S. T., Park, J., \& Ram, S. (2004). Design science in information systems research. MIS Quarterly, 28(1), 75105.

Ingalls, R. G. (2008, 2008). Introduction to simulation. Paper presented at the Proceedings of the 40th Conference on Winter Simulation.

Ives, B., Olson, M. H., \& Baroudi, J. J. (1983). The measurement of user information satisfaction. Communications of the ACM, 26(10), 785-793.

Keller, C. (2009). User Acceptance of Virtual Learning Environments: A Case Study from Three Northern European Universities. Communications of the Association for Information Systems, 25.

Lange, C. F. (2006). Improving the quality of UML models in practice. Paper presented at the Proceedings of the 28th international conference on Software engineering.

Lindland, O. I., Sindre, G., \& Solvberg, A. (1994). Understanding quality in conceptual modeling. Software, IEEE, 11(2), $42-49$.

Liu, S., Liu, Y., André, E., Choppy, C., Sun, J., Wadhwa, B., \& Dong, J. S. (2013). A formal semantics for complete UML state machines with communications. Paper presented at the Integrated Formal Methods.

Merseguer, J., Campos, J., Bernardi, S., \& Donatelli, S. (2002). A compositional semantics for UML state machines aimed at performance evaluation. Paper presented at the Discrete Event Systems, 2002. Proceedings. Sixth International Workshop on.

Nelson, H. J., Poels, G., Genero, M., \& Piattini, M. (2012). A conceptual modeling quality framework. Software Quality Journal, 20(1), 201-228. doi: 10.1007/s11219-011-9136-9

Otero, M. C., \& Dolado, J. J. (2004). Evaluation of the comprehension of the dynamic modeling in UML. Information and Software Technology, 46(1), 35-53.

Pastor, O., Gómez, J., Insfrán, E., \& Pelechano, V. (2001). The OO-Method approach for information systems modeling: from objectoriented conceptual modeling to automated programming. Information Systems, 26(7), 507-534.

Poelmans, S., \& Wessa, P. (2013). A Constructivist Approach in an e-Learning Environment for Statistics. Interactive Learning Environments (accepted).

Reggio, G., Leotta, M., Ricca, F., \& Clerissi, D. (2013). What are the used UML diagrams? A Preliminary Survey. Paper presented at the EESSMOD@ MoDELS.

Schenk, K. D., Vitalari, N. P., \& Davis, K. S. (1998). Differences between Novice and Expert Systems Analysts: What Do We Know and What Do We Do? Journal of Management Information Systems, 15(1), 9-50. doi: 10.2307/40398371

Sedrakyan, G. (2016). Process-oriented feedback perspectives based on feedback-enabled simulation and learning process data analytics. (PhD thesis), KU Leuven.

Sedrakyan, G., De Weerdt, J., \& Snoeck, M. (2016). Process-mining enabled feedback: “tell me what I did wrong” vs. "tell me how to do it right". Computers in Human Behavior, 57(C), 352-376. doi: 10.1016/j.chb.2014.09.054

Sedrakyan, G., \& Snoeck, M. (2012). Technology-enhanced support for learning conceptual modeling Enterprise, Business-Process and Information Systems Modeling (pp. 435-449): Springer.

Sedrakyan, G., \& Snoeck, M. (2013a). Feedback-enabled MDA-prototyping effects on modeling knowledge Enterprise, BusinessProcess and Information Systems Modeling (pp. 411-425): Springer.

Sedrakyan, G., \& Snoeck, M. (2013b). A PIM-to-Code requirements engineering framework. Paper presented at the Proceedings of Modelsward 2013-1st International Conference on Model-driven Engineering and Software Development-Proceedings.

Sedrakyan, G., \& Snoeck, M. (2014a). Do we need to teach testing skills in courses on requirements engineering and modelling? Paper presented at the CEUR Workshop Proceedings.

Sedrakyan, G., \& Snoeck, M. (2014b). Lightweight semantic prototyper for conceptual modeling Advances in Conceptual Modeling (pp. 298-302): Springer.

Sedrakyan, G., \& Snoeck, M. (2015). Effects of Simulation on Novices' Understanding of the Concept of Inheritance in Conceptual Modeling Advances in Conceptual Modeling (pp. 327-336): Springer.

Sedrakyan, G., \& Snoeck, M. (2016). Enriching model execution with feedback to support testing of semantic conformance between models and requirements: Design and evaluation of feedback automation architecture. Paper presented at the Modelsward 2016 - 4th International Conference on Model-driven Engineering and Software Development, Rome, Italy.

Sedrakyan, G., Snoeck, M., \& De Weerdt, J. (2014). Process Mining Analysis of Conceptual Modeling Behavior of Novices empirical study using JMermaid modeling and experimental logging environment. Computers in Human Behavior, 41(C), 486-503. doi: 10.1016/j.chb.2014.09.054

Sedrakyan, G., Snoeck, M., \& Poelmans, S. (2014). Assessing the effectiveness of feedback enabled simulation in teaching conceptual modeling. Computers \& Education, 78, 367-382.

Segars, A. H., \& Grover, V. (1993). Re-examining perceived ease of use and usefulness. MIS Quarterly, 17(4), 517-525.

Siau, K., \& Cao, Q. (2001). Unified Modeling Language (UML)-a complexity analysis. Journal of Database Management, 12 (1), 26.

Siau, K., Erickson, J., \& Lee, L. Y. (2005). Theoretical vs. practical complexity: The case of UML. Journal of Database Management, $16(3), 40$.

Snoeck, M. (2014). Enterprise Information Systems Engineering: The MERODE Approach: Springer.

Snoeck, M., \& Dedene, G. (1998). Existence Dependency: The key to semantic integrity between structural and behavioral aspects of object types. Software Engineering, IEEE Transactions on, 24(4), 233-251. 
Snoeck, M., Haesen, R., Buelens, H., De Backer, M., \& Monsieur, G. (2007). Computer Aided Modelling Exercises. Informatics in Education, 6(1), 231-248.

Snoeck, M., Michiels, C., \& Dedene, G. (2003). Consistency by construction: the case of MERODE Conceptual Modeling for Novel Application Domains (pp. 105-117): Springer.

Trochim, W. M. (2000). The Research Methods Knowledge Base, Internet WWW page. http://www.socialresearchmethods.net/kb/index.php; Version current as of September 2015.

Venkatesh, V., Morris, M. G., Davis, G. B., \& Davis, F. D. (2003). User acceptance of information technology: Toward a unified view. MIS Quarterly, 27(3).

Wilmont, I., Hengeveld, S., Barendsen, E., \& Hoppenbrouwers, S. (2013). Cognitive Mechanisms of Conceptual Modelling. In W. Ng, V. Storey, \& J. Trujillo (Eds.), Conceptual Modeling (Vol. 8217, pp. 74-87): Springer Berlin Heidelberg.

Wixom, B. H., \& Todd, P. A. (2005). A theoretical integration of user satisfaction and technology acceptance. Information systems research, 16(1), 85-102.

Yang, M. C. (2005). A study of prototypes, design activity, and design outcome. Design Studies, 26(6), 649-669. 


\section{Appendix 1: Experiment cases}

*ADVICE: To make it easier to answer the questions trace the scenarios by creating new object instances for each scenario appended with scenario identifier, e.g. when creating objects of type P for question 1, name them p1-q1, p2-q1,etc.

\section{ASSUMPTIONS:}

1. When a scenario is given, we assume that the events are processed without any other events in between. For example, if a scenario says cr_a, cr_y, v, then no other events are processed in between cr_a and cr_y and between cr_y and v.

2. To avoid confusions, for each scenario we assume that new object instances are created. For each scenario object instances created for that specific scenario are used. For example, if a scenario1 says: cr_a, cr_y, v

a) Create object of type A named a-scenarioIdentifier, e.g. a-q1

b) Create object of type $Y$ named y-scenarioIdentifier AND as a master object choose a-scenarioIdentifier (do not use an A object used in other executed scenario), e.g. y-q1 related to a-q1

c) next the execution of event $v(\mathrm{O} / \mathrm{M}$ of $\mathrm{Y}$ object $)$ should use the object $\mathrm{y}-\mathrm{q} 1$

\section{Case 1: PAXY}

Indicate if the following execution scenarios are supported (TRUE/FALSE) given the class diagram, statecharts and interaction model below. Please provide a short explanation for the reason of your choice.
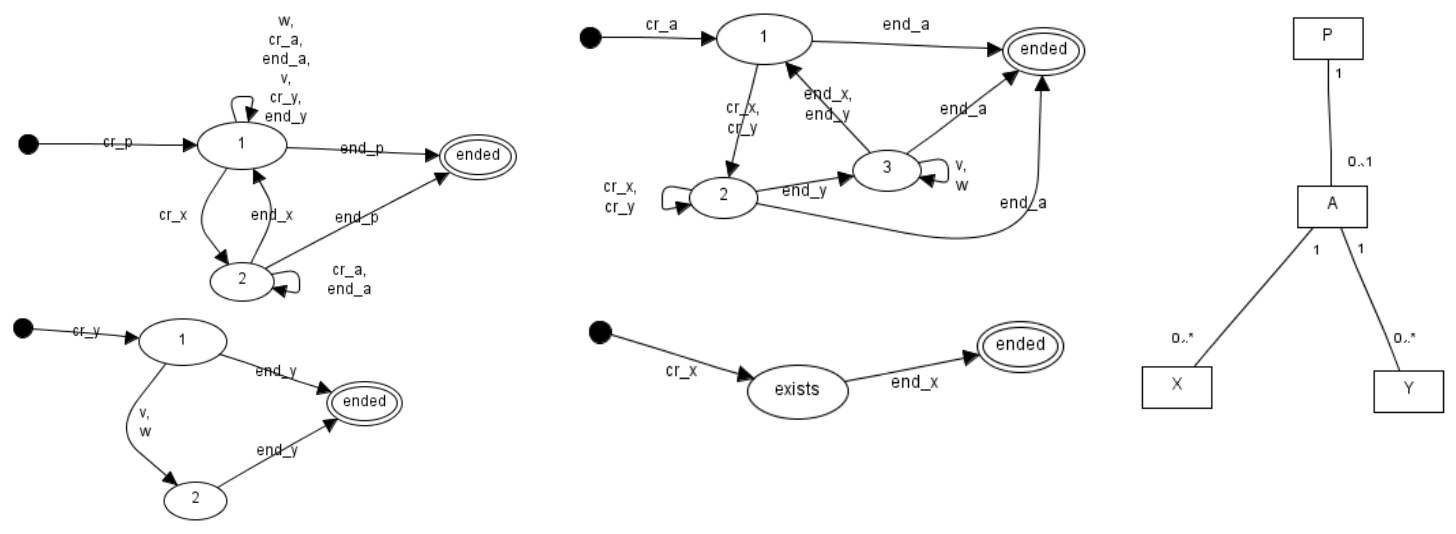

\section{QUESTIONS (execution scenarios):}

1) Having created one object of type P (p1-q1) and one object of type A (a1-q1), registering $X$ and $Y$ objects can be done in any order: both the sequence of events cr_x (x1-q2).cr_y (y1-q1) and cr_y(y2-q1). cr_x (x2-q1) are POSSIBLE.

2) An object of type A (a-q2) related to the object of type P (p-q2) CANNOT have an arbitrary number of objects of type X. For instance for the object a-q2 one CANNOT create objects x1-q2, x2-q2, x3-q2 each of type $\mathrm{X}$ through a sequence of cr_x events.

3) Having created one object of type P (p-q3), one object of type A (a-q3) and one object of type $Y$ (y-q3), an object of type Y (yq3) can move then from state 1 to state "ended" by means of a sequence of events $v$, w, end_y.

4) Having created one object of type $P(p-q 4)$, one object of type A (a-q4), the object of type $P(p-q 4)$ can move from state 1 to state "ended" by means of a sequence of cr_y (y-q4), end_y (y-q4), end_p (p-q4)

5) An object of type A (a-q5) related to the object of type P(p-q5) can move from state 1 to state "ended" by means of a sequence of cr_x (x-q5), cr_y (y-q5), end_y (y-q5), end_x (x-q5), end_a (a-q5)

6) An object of type P (p-q6) can move from state 1 to state "ended" by means of a sequence of cr_a (a-q6), cr_y (y-q6), v (y-q6), end_y (y-q6), end_a (a-q6), end_p (p-q6)

7) An object of type $P$ (p-q7) can move from state 1 to state "ended" by means of a sequence of cr_a (a-q7), cr_x (x-q7), end_x (xq7), end_a (a-q7), end_p (p-q7)

8) An object of type P (p-q8) can move from state 1 to state "ended" by means of a sequence of cr_a(a1-q8), cr_x (x-q8), cr_a (a2$\mathrm{q} 8)$, end_x (x-q8), end_a(a1-q8), end_a (a2-q8), end_p(p-q8)

9) An object of type P (p-q9) can move from the initial state to state "ended" by means of a sequence of cr_p (p-q9), cr_a (a-q9), cr_x (x-q9), end_a (a-q9), end_x (x-q9), end_p (p-q9)

10) Each A-object that has an $X$-object, needs at least one $Y$-object to successfully complete its lifecycle. 


\section{Case 2: ABCD}

Indicate if the following execution scenarios are supported (TRUE/FALSE) given the class diagram, statecharts and interaction model below. Please provide a short explanation for the reason of your choice.
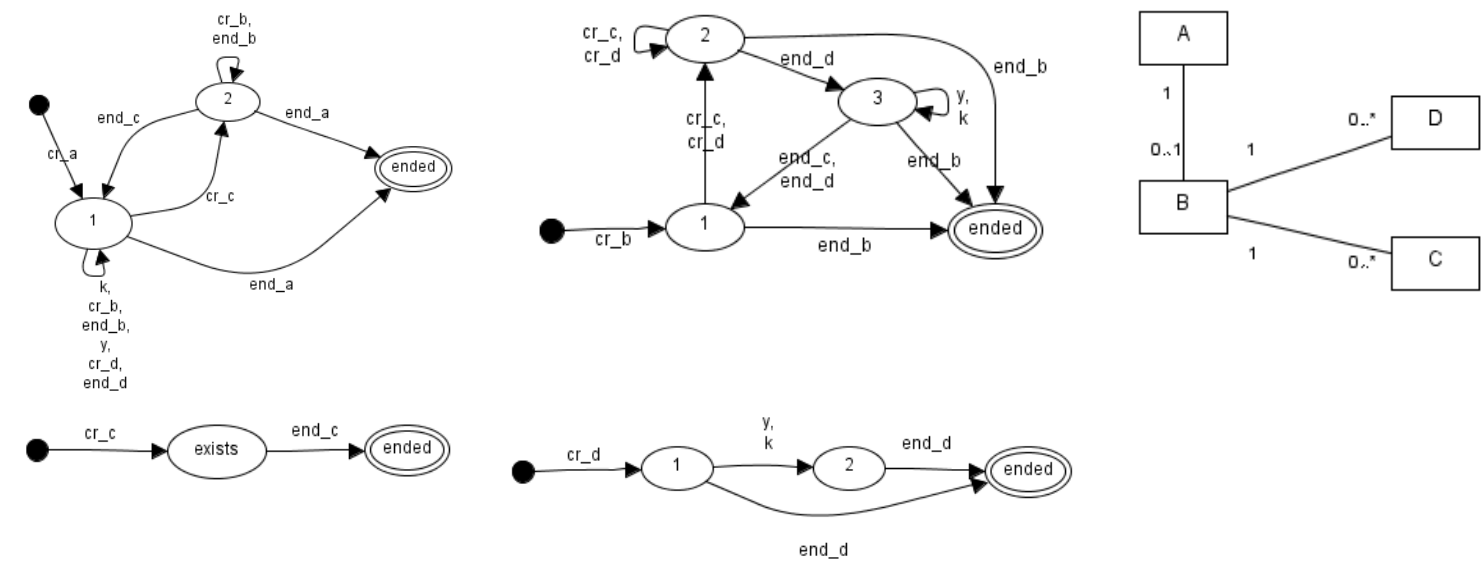

\section{QUESTIONS (execution scenarios):}

1) Having created one object of type A (a1-q1) and one object of type B (b1-q1), it is possible to register C and D objects in any order : both the sequence of events cr_c (c1-q2).cr_d (d1-q1) AND cr_d(d2-q1). cr_c (c2-q1) are possible

2) Having created one object of type A (a-q2), one object of type B (b-q2) and one object of type D (d-q2), an object of type D (dq2) can move then from state 1 to state "ended" by means of a sequence of events $y$, $k$, end_d.

3) An object of type B (b-q3) related to the object of type A (a-q3) can have an arbitrary number of objects of type C. For instance for the object b-q3 one can create objects c1-q3, c2-q3, c3-q3 each of type $\mathrm{C}$ through a sequence of cr_c events.

4) Having created one object of type A (a-q4), one object of type B (b-q4) it is IMPOSSIBLE for object A (a-q4) to move from state 1 to state "ended" by means of a sequence of cr_d (d-q4), end_d (d-q4), end_a (a-q4)

5) An object of type B (b-q5) related to the object of type A(a-q5) CANNOT move from state 1 to state "ended" by means of a sequence of cr_c (c-q5), cr_d (d-q5), end_d (d-q5), end_c (c-q5), end_b (b-q5)

6) An object of type A (a-q6) can move from state 1 to state "ended" by means of a sequence of cr_b (b-q6), cr_c (c-q6), end_c (cq6), end_b (b-q6), end_a (a-q6)

7) An object of type A (a-q7) can move from state 1 to state "ended" by means of a sequence of cr_b (b-q7), cr_d (d-q7), k (d-q7), end_d (d-q7), end_b (b-q7), end_a (a-q7)

8) An object of type A (a-q8) can move from state 1 to state "ended" by means of a sequence of cr_b (b-q8), cr_c (c-q8), cr_b (b2q8), end_c (c-q8), end_b (b-q8), end_b (b2-q8), end_a (a-q8)

9) An object of type A (a-q9) cannot move from the initial state to state "ended" by means of a sequence of cr_a (a-q9), cr_b (b-q9), cr_c (c-q9), end_b (b-q9), end_c (c-q9), end_a (a-q9)

10) Each B-object that has a D-object, needs at least one other D object or C-object to successfully complete its lifecycle. 


\section{Appendix 2: Evaluation questionnaire}

1 = totally disagree; 2 = disagree; 3 = disagree somewhat; $4=$ agree somewhat; $5=$ agree; $6=$ totally agree

\begin{tabular}{|c|c|c|c|c|c|c|}
\hline I find the prototype easy to use. & $\mathrm{O} 1$ & $\mathrm{O} 2$ & O 3 & $\mathrm{O} 4$ & O 5 & O 6 \\
\hline It was easy to learn and understand the prototype. & $\mathrm{O} 1$ & $\mathrm{O} 2$ & $\mathrm{O} 3$ & $\mathrm{O} 4$ & $\mathrm{O} 5$ & O 6 \\
\hline I can without effort get the prototype to do what I want it to do. & $\mathrm{O} 1$ & $\mathrm{O} 2$ & $\mathrm{O} 3$ & $\mathrm{O} 4$ & $\mathrm{O} 5$ & O 6 \\
\hline $\begin{array}{l}\text { Using the prototype improves my understanding of behavior modeling } \\
\text { and statecharts. }\end{array}$ & $\mathrm{O} 1$ & $\mathrm{O} 2$ & O 3 & $\mathrm{O} 4$ & O 5 & O 6 \\
\hline $\begin{array}{l}\text { Using the prototype makes me understand behavior modeling and } \\
\text { statecharts much faster. }\end{array}$ & $\mathrm{O} 1$ & $\mathrm{O} 2$ & O 3 & $\mathrm{O} 4$ & O 5 & O 6 \\
\hline $\begin{array}{l}\text { Using the prototype enables me to remove my uncertainty about the } \\
\text { presented behavior model/statecharts. }\end{array}$ & $\mathrm{O} 1$ & $\mathrm{O} 2$ & O 3 & $\mathrm{O} 4$ & O 5 & O 6 \\
\hline $\begin{array}{l}\text { If had the choice, or opportunity I would use prototyping to learn } \\
\text { behavior modeling. }\end{array}$ & $\mathrm{O} 1$ & $\mathrm{O} 2$ & O 3 & $\mathrm{O} 4$ & O 5 & O 6 \\
\hline $\begin{array}{l}\text { If I had to vote, I would vote in favour of using prototyping in the } \\
\text { classroom }\end{array}$ & $\mathrm{O} 1$ & $\mathrm{O} 2$ & O 3 & $\mathrm{O} 4$ & O 5 & O 6 \\
\hline I am enthusiastic about using the prototyping in this kind of courses & $\mathrm{O} 1$ & $\mathrm{O} 2$ & $\mathrm{O} 3$ & $\mathrm{O} 4$ & O 5 & O 6 \\
\hline Overall, I am very satisfied with using the prototype & $\mathrm{O} 1$ & $\mathrm{O} 2$ & $\mathrm{O} 3$ & $\mathrm{O} 4$ & $\mathrm{O} 5$ & $\mathrm{O} 6$ \\
\hline My experience with the prototype meets my general expectations & $\mathrm{O} 1$ & $\mathrm{O} 2$ & $\mathrm{O} 3$ & $\mathrm{O} 4$ & O 5 & O 6 \\
\hline Using the prototype was a positive experience & $\mathrm{O} 1$ & $\mathrm{O} 2$ & $\mathrm{O} 3$ & $\mathrm{O} 4$ & $\mathrm{O} 5$ & $\mathrm{O} 6$ \\
\hline Automated feedback helps to interpret the model better & $\mathrm{O} 1$ & $\mathrm{O} 2$ & $\mathrm{O} 3$ & $\mathrm{O} 4$ & O 5 & O 6 \\
\hline
\end{tabular}

\section{Previous knowledge ( in terms of having behavior / statecharts modeling and/or programming course(s) before )}

1 = no knowledge/experience at all; 2 = little knowledge ( a few hours course ); 3 = moderate knowledge ( intermediate level course ); 4 = extensive knowledge ( advanced course $(s)$ )

\begin{tabular}{|l|llll|}
\hline $\begin{array}{l}\text { Previous knowledge on behavior modeling / statecharts in previous } \\
\text { degree }\end{array}$ & O 1 & O 2 & O 3 & O 4 \\
\hline Previous knowledge on programming in previous degree & O 1 & O 2 & O 3 & O 4 \\
\hline Previous knowledge on testing a software in previous degree & O 1 & O 2 & O 3 & O 4 \\
\hline
\end{tabular}


I could use a new software application well ...

$1=$ not at all confident; 2 = probably not; 3 = rather not; 4 = rather yes; 5 = likely yes; $6=$ Totally Confident: Yes

\begin{tabular}{|l|llllll|}
\hline$\ldots$ even if I had never used an application like it before. & O 1 & O 2 & O 3 & O 4 & O 5 & O 6 \\
\hline$\ldots$ if I had just the built-in-help facility or manual for assistance. & O 1 & O 2 & O 3 & O 4 & O 5 & O 6 \\
\hline ... if I had first seen someone else using it before trying it myself. & O 1 & O 2 & O 3 & O 4 & O 5 & O 6 \\
\hline $\begin{array}{l}\text {... using only the internet for assistance. } \\
\text { O O 1 }\end{array}$ & O 2 & O 3 & O 4 & O 5 & O 6 \\
\hline
\end{tabular}

On Average, I use computers (laptop, desktop, tablet) per day:
less than one hour
one to two hours
three to five hours
six to eight hours
eight or more hours 